\title{
Systematic Reviews and Meta-Analyses in Neuroepidemiological Research: Applications and Issues
}

Meta-analyses have been particularly successful in the last few years in several fields of medicine including neurology. Summarizing the evidence in a comprehensive and systematic manner, whilst increasing the overall statistical power, are two features that account for the attraction of meta-analysis in health-related and other types of research fields. The need for more meta-analyses may be determined by another problem particularly prominent in neurology: the scatter of research. It has been reported that neurology is the most scattered discipline in medicine with nearly 3,000 studies relating to neurological diseases being published in almost 900 different journals. Keeping up to date with new research is therefore particularly challenging for the neurologist. We believe that summarizing the evidence from neuroepidemiological studies in a comprehensive manner with meta-analyses is an important step in the right direction in order to translate research evidence into clinical practice.

In this special issue of Neuroepidemiology, we have included meta-analyses of both 'classical' and 'emerging' topics that should be of interest to neuroepidemiologists. Of the six papers included, five are related to the application of meta-analyses in substantive clinical research fields and one concerns the key methodological developments specific to the conduct of meta-analyses.

The paper by Bharucha et al. assesses the issues in the pooling of descriptive studies of epilepsy that may be useful in order to determine the overall burden of this disease in low- and middle-income countries. Fiest et al. describe the application of meta-analyses to incidence and prevalence studies and describe how the meta-analyses of these types of studies can facilitate the prioritization of future research and have a direct influence on health systems and services. Biological markers are becoming increasingly important in all areas of epidemiology. The report by Takeda et al. presents an example of the utility of metaanalyses in identifying potential biomarkers that may be relevant to neurodegenerative diseases such as Parkinson's disease. In their report the authors present information for the first time on the association between blood levels or dietary intakes of vitamin A and carotenoids and risk of Parkinson's disease. Genetics can play an important role in neurological diseases and van der Mei et al. discuss how meta-analysis can be used to investigate gene-environment interactions with examples from the neuroepidemiological literature. The role of alternative therapies as potentially useful interventions in neurology are poorly reported and have seldom been systematically reviewed. Zhang et al. summarise and critique the current evidence on the role of acupuncture as a useful intervention for stroke and stroke-related disorders. Finally, Copetti et al. bring together the evidence on recent methodological developments related to the conduct of metaanalyses.

Several major themes re-occur through all of these reports that are fundamental to the robust and reliable con- 
duct of systematic reviews and meta-analyses. First, the aim of a meta-analysis is to investigate and explain potential sources of heterogeneity in order to identify specific subpopulations that may be harmed or experience a benefit from the same exposure or intervention. Second, because of some features of many neurological diseases (such as low frequency and phenotypic heterogeneity), many published studies in neurology are small. Metaanalyses may therefore be an essential tool to increase statistical power in order to detect modest effect sizes. Third, the overall quality and reliability of the meta-analysis is dependent on the quality and methodological rigour of the individual studies included. Fourth, combining evidence from both observational studies and clinical trials needs to be done carefully due to the inherent biases in observational studies. A simple pooling of results from these two different sources of evidence could be misleading. Finally, collaborative research in the form of individual participant data meta-analyses (that are challenging to conduct as they require harmonization of source data from individual studies that have used different measures and definitions), is likely to be the way forward in order to address unanswered important research questions in neuroepidemiology.

We would like to thank Dr. Yoshiro Kokubo for reviewing some of the draft manuscripts and all of the authors for their contributions, and hope that readers will find these reports as interesting and informative as we have.

Giancarlo Logroscino, Bari Derrick A. Bennett, Oxford 